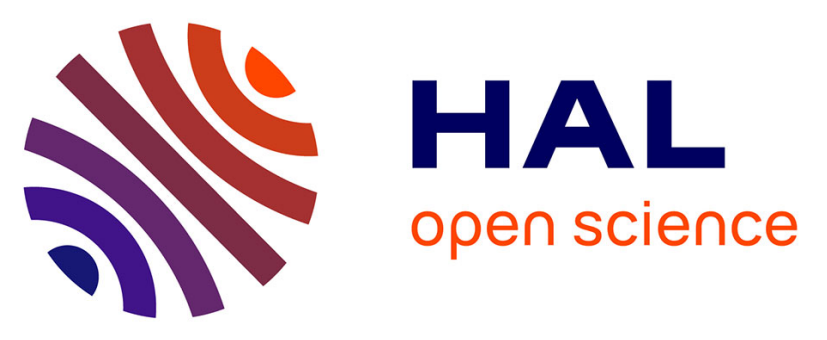

\title{
Multicontrast MRI-based radiomics for the prediction of pathological complete response to neoadjuvant chemotherapy in patients with early triple negative breast cancer
}

\author{
Angeline Nemeth, Pierre Chaudet, Benjamin Leporq, Pierre-Etienne Heudel, \\ Fanny Barabas, Olivier Tredan, Isabelle Treilleux, Agnès Coulon, Frank \\ Pilleul, Olivier Beuf
}

\section{- To cite this version:}

Angeline Nemeth, Pierre Chaudet, Benjamin Leporq, Pierre-Etienne Heudel, Fanny Barabas, et al.. Multicontrast MRI-based radiomics for the prediction of pathological complete response to neoadjuvant chemotherapy in patients with early triple negative breast cancer. Magnetic Resonance Materials in Physics, Biology and Medicine, 2021, 10.1007/s10334-021-00941-0 . hal-03408526

\author{
HAL Id: hal-03408526 \\ https://hal.science/hal-03408526
}

Submitted on 29 Oct 2021

HAL is a multi-disciplinary open access archive for the deposit and dissemination of scientific research documents, whether they are published or not. The documents may come from teaching and research institutions in France or abroad, or from public or private research centers.
L'archive ouverte pluridisciplinaire HAL, est destinée au dépôt et à la diffusion de documents scientifiques de niveau recherche, publiés ou non, émanant des établissements d'enseignement et de recherche français ou étrangers, des laboratoires publics ou privés. 
Title page

3 Multicontrast MRI-based radiomics for the prediction of pathological complete

4 response to neoadjuvant chemotherapy in patients with early triple negative breast

5 cancer

6 Authors:

7 Angeline NEMETH, PhD,1 Pierre CHAUDET, MD,2 Benjamin LEPORQ, PhD,1 Pierre-Etienne 8 HEUDEL, MD,3 Fanny BARABAS,2 Olivier TREDAN, MD-PhD,3 Isabelle TREILLEUX, MD-

$9 \mathrm{PhD}, 4$ Agnès COULON, MD,2 Frank PILLEUL, MD-PhD,1,2 and Olivier BEUF, PhD, 1

10 Author Affiliations:

11 1Univ Lyon, INSA-Lyon, Université Claude Bernard Lyon 1, UJM-Saint Etienne, CNRS, Inserm, CREATIS UMR 5220, U1206, F69621, Lyon, France

13 2Department of Radiology, Centre Léon Bérard, Lyon, France

14 3Department of Medical Oncology, Centre Léon Bérard, Lyon, France

15 4Department of Pathology, Centre Leon Bérard, Lyon, France

16 Corresponding author:

Benjamin Leporq, +33469856258

Benjamin.leporq@creatis.insa-lyon.fr

Centre Léon Bérard, 28 Prom. Léa et Napoléon Bullukian, 69008 Lyon, France

$\underline{\text { Acknowledgments }}$

22 This study was conducted as part of the LABEX PRIMES (ANR-11-LABX-0063) of the

23 "Université de Lyon", within the "Investissements d'Avenir" program (ANR-11-IDEX-

24 0007) operated by the French National Research Agency (ANR). This study was also 25 supported by the SIRIC LyriCAN grant (INCa_INSERM_DGOS_12563). We thank Sophie 26 Darnis for her help with English language editing. 
Introduction:

To assess pre-therapeutic MRI-based radiomic analysis to predict the pathological complete response to neoadjuvant chemotherapy (NAC) in women with early triple negative breast cancer (TN).

32 Materials and methods:

This monocentric retrospective study included $75 \mathrm{TN}$ female patients with MRI (T1-weighted, T2weighted, diffusion-weighted and dynamic contrast enhancement images) performed before NAC. For each patient, the tumor(s) and the parenchyma were independently segmented and analyzed with radiomic analysis to extract shape, size, and texture features. Several sets of features were realized based on the 4 different sequence images. Performances of 4 classifiers (random forest, multilayer perceptron, support vector machine (SVM) with linear or quadratic kernel) were compared based on pathological complete response (defined on the excised tissues), on 100 draws with $75 \%$ as training set and $25 \%$ as test.

Results:

The combination of features extracted from different MR images improved the classifier performance (more precisely, the features from T1W, T2W and DWI). The SVM with quadratic kernel showed the best performance with a mean AUC of 0.83 , a sensitivity of 0.85 and a specificity of 0.75 in the test set.

Conclusion:

MRI-based radiomics may be relevant to predict NAC response in TN cancer. Our results promote the use of multi-contrast MRI sources for radiomics, providing enrich source of information to enhance model generalization.

$\underline{\text { Keywords }}$

52 Breast Cancer; Radiomics; Multi-contrast MRI; Triple Negative Breast Cancer.

$\underline{\text { Abbreviations }}$

- $\quad$ AUC: Area Under the Curve ROC

- DCE: dynamic contrast enhancement 
- HER: Human Epidermal Growth Factor

58 - VOI: volume of interest

59 - MLP: multilayer perceptron

60 MRI: magnetic resonance imaging

61 - NAC: neo-adjuvant chemotherapy

62 - $\mathrm{pCR}$ : pathological complete response

63 - ROC: receiver operating characteristic

64 - SVM: support vector machine

$65-\underline{T N}$ : triple negative

66 - TNM: tumor, node, metastases

67 - T1W: T1-weighted imaging

68 - $\underline{T} 2 \mathrm{~W}:$ T2-weighted imaging

69 - DWI: diffusion weighted imaging

70 - GLCM: Gray-level co-occurrence matrix

71 - GLSZM: Gray-level size zone matrix

72 - NGTDM: Neighborhood gray tone difference matrix

73 - $\quad$ SURF: Speed-Up Robust Features

74 - $\quad$ SUB3: subtraction between the image 3 min post-injection and the image pre-injection 
INTRODUCTION

Breast cancer is the most frequently diagnosed cancer in women with $2,090,000$ new cases and 627,000 deaths worldwide in 2018 [1]. Among the different types of breast cancer, triple negative (TN) cancer is characterized by estrogen and progesterone receptor level lower than $10 \%$, and an absence of over-expression of HER-2 (Human Epidermal Growth Factor Receptor-2). TN cancers account for 10 to $24 \%$ of all breast cancers, and 57 to $88 \%$ of cancers with BRCAl mutation in women [2]. TN tumors are generally larger, diagnosed at the highest grade, and associated with worse prognosis [3]. At early stage, patients with $\mathrm{TN}$ cancer receive systemic treatments generally limited to cytotoxic chemotherapy, no targeted therapies are currently proposed. Neoadjuvant chemotherapy (NAC) treatment are used: i) to reduce the initial tumor volume in order to allow a conservative surgical treatment; ii) to better eradicate the micrometastatic disease; iii) to assess tumor chemosensibility to determine the most appropriate adjuvant treatments [4]. The pathological complete response (pCR) to NAC is an important prognostic factor for the disease-free survival and overall survival in breast cancer $[5,6]$. The efficacy of NAC varies according to the tumor's genetic profile and pCR levels from 10 to 50\% [7]. Therefore, there is a clinical need to identify patients who will not respond to NAC to eventually direct them to alternative therapeutic strategies.

According to the latest EUSOBI recommendations [8], pre- and post-chemotherapy MRI examination should be performed in women with early breast cancer receiving NAC. Since 2012 [9], a new discipline called "radiomics" has drawn increasing attention in cancer research in disease detection, diagnosis, and prediction of treatment response, and several studies investigated prediction of pCR to breast cancer chemotherapy [10-14]. Radiomics is based on the hypothesis that genetics, molecular, cellular and tissular modifications can be observed on images $[15,16]$. Technically, radiomics consist in extracting a high number of quantitative parameters from radiologic images in order to determine their relationships with the underlying pathophysiology $[17,18]$. Moreover, through functional and anatomical information that MRI provided with on whole tissues, MRI-based radiomics allows to access to quantitative information refining the entire tumor and its micro-environment, and to probe tumor heterogeneity [19]. Some radiomic studies focused not only on the tumor, but also on the surrounding mammary parenchyma. The tumor microenvironment is known to partly contribute to the 

be as important as the tumor analysis itself [14]. In this study, we explored the performance of radiomics in tumor and ipsilateral parenchymal mammary MRI to predict the pCR to NAC in TN breast cancer female patients.

\section{METHOD}

\section{Study design}

111 Patients enrolled in this retrospective study underwent a pre-therapeutic MRI protocol between 112 January 2008 and Jun 2017 in the French comprehensive cancer center "Centre Léon Bérard". All 113 patients had an early triple negative breast cancer (i.e. without metastasis) and were treated with 114 neoadjuvant chemotherapy (NAC) before a surgical treatment. All women received a sequential NAC 115 therapy based on anthracyclines-cyclophosphamide, then taxanes. Data were excluded for: i) poor 116 quality of MRI imaging (moving artifacts, missing of some images); ii) NAC treatment initiated prior 117 to MRI. This retrospective study was approved by our institutional review board and the requirement 118 to obtained informed consent was waived.

\section{Pre-therapeutic MRI protocol}

121 All breast MRI examinations were performed at Leon Berard center with patient in prone position 122 using a 1.5T Achieva system (Philips Healthcare, Best, Netherlands) and with the use of a dedicated 123 seven-channel or sixteen-channel breast surface coil (SENSE-Breast-7 or SENSE-Breast16M). The 124 pre-therapeutic MRI protocol was sequentially composed of a T1-weighted imaging, a T2-weigthed imaging, a diffusion weighted imaging and a dynamic contrast enhanced imaging (Figure 1). MRI

126 protocol parameters are summarized in Table 1. Dynamic contrast enhancement (DCE) MR imaging 127 was acquired after intravenous injection of $0.1 \mathrm{mmol} / \mathrm{kg}$ gadolinium-based contrast agent. Images 128 noted "SUB3" were obtained subtracting images acquired 3 min after the injection and those acquired 129 before injection using DCE-MRI.

\section{Segmentation of volume of interest}

132 The 3D volume of interest (VOI) was delineated manually using itk-SNAP software 
133 (www.itksnap.org) on few slices of SUB3 images; the inter-slice interpolation option was used to

134 complete the mask between slices to have a 3D volume. More precisely, for each patient, tumor and 135 parenchyma were initially segmented by a radiologist intern PC, after correcting by a senior 136 radiologist (more than 10 years of experience in breast imaging AC). In the case of multiple lesions, 137 each of them was considered independently with separate 3D binary mask. The delineation of the 138 parenchyma included the fibroglandular tissue and the adipose tissue and excluded the skin and the 139 tumor. The segmentations of tumor and parenchyma resulting from the consensus of the two 140 radiologists $\mathrm{PC}$ and $\mathrm{AC}$ were then used for the extraction of size, shape, intensity distribution, and 141 other texture features. Areas impacted by the presence of a clip were excluded from the VOI for the 142 computation of intensity distribution and texture features (but not for shape and size features). An 143 affine transformation was used to reposition VOIs segmented on SUB3 images on the other images 144 (T1W, T2W and DWI) using matrix dimension and patient position information. A third expert FB 145 was asked to delineate a subset of twenty-four tumors in order to analyze the variability of 146 segmentations with the difficulty to have any access to the assessment of the other two radiologists 147 and without access to the patient file.

\section{Features extraction}

150 We tested multiple configurations. We first use only SUB3 images with features extracted from lesion 151 and parenchyma, and progressively include features from T1-weitghted images, T2-weitghted images, 152 and DW images in the feature set. Extraction of feature set was performed with MATLAB 2019a (The 153 Mathworks, Natick, MA, USA) using an in-house software. 3D-image intensities were filtered using 154 Collewet method [21]. The initial feature set of a ROI was composed of 342 features: 96 shape and 155 size characteristics, 14 intensity distribution characteristics, and 232 textural characteristics. As shown 156 in the Figure 2, size and shape features were directly extracted from the binary masks and were based 157 on morphological skeletonization and distance transforms, affine moment invariants [22], Hu moment 158 [23], Zernike moment [24, 25], and conventional metrics. Intensity distribution features were extracted 159 from masked MR images from the histogram built with 256 bins (14 features: average, standard 160 deviation, full width at half maximum, variance, minimum, maximum, range, interquartile range, 161 kurtosis, skewness, entropy energy, root mean square, mean deviation, median deviation). Before the 
extraction of texture features, voxels were isotropically resampled using an affine transformation and a nearest-neighbor interpolation and then discretized in a smaller number of gray levels. This operation was done using an equal probability algorithm to define decision thresholds in the volume such as the number of voxels for a given reconstructed level is the same in the quantized volume for all gray levels. Images were discretized in 8, 16, 24, 32, 48 and 64 grey levels and for each level four matrix were built: GLCM (Gray-level co-occurrence matrix) ( $\mathrm{n}=21)$, GLRLM (Gray-level run length matrix) $(n=13)$, GLSZM (Gray-level size zone matrix) $(n=13)$ and NGTDM (Neighborhood gray tone difference matrix) (n=5) from which characteristics were extracted. Frequency domain-based texture features were extracted from the Gabor filters responses and from features extracted from image spectrum after 2D discrete Fourier transform. GLCM and GLRLM will be computed for 4 directions $\left(0^{\circ}, 45^{\circ}, 90^{\circ}\right.$ and $\left.135^{\circ}\right)$ with an offset of 1 pixel. For GLSZM and NGTDM, a 26-pixel connectivity will be used. For Gabor filtering, 5 scales, 6 orientations, and a minimal wavelength of 3 were used. Other texture feature based on images primitive were also extracted using different detectors and descriptors (such as Speed-Up Robust Features (SURF) detector, Local Oriented Statistics Information Booster (LOSIB) descriptor, Harris detector); lacunarity computation or quad tree decomposition. These features were already described in previous studies $[10,11,26]$. For multiparametric case, only textural and intensity features (set of 492 features) were computed on the other imaging (T1W, T2W and DWI) and added to the initial feature set as illustrated in Figure 2.

\section{Data mining}

The test set included $25 \%$ of the total number of tumors, randomly selected from the whole data set, with a balance between pCR and non-pCR. One hundred different configurations of the training and test sets were used. Z-score normalization was applied on each features of the feature set. Then, a dimension reduction was applied using ReliefF method [27] to select the twenty most relevant features on the whole data set. From the reduced feature set, supervised machine learning was used to build the prediction model. Four classifiers were evaluated: a multilayer perceptron (MLP) trained with a stochastic gradient algorithm using an adaptive learning rate and a regularization of the synaptic weigths ( $\square=0.1,5$ mini-batches, 30 hidden nodes, and 60 epochs); a support vector machine (SVM) with a linear kernel; a SVM with a quadratic kernel (trained with box-constraint $\mathrm{c}=1$ ) and a random 
forest ( 3 splits and 50 learning cycles). The difference between the AUC for the training set and for the test set was used to evaluate the overfitting of the classification method. We evaluated the performance of the classification models thanks to the area under the curve (AUC) of the receiver operating characteristic (ROC). The difference in AUC between the training and the testing sets was used as an indicator for the predictive model to generalize the estimation.

\section{Statistical Analysis}

198 A Sørensen-Dice index was computed to compared the delineation given by various radiologists. An intraclass correlation coefficient (ICC) for each radiomic features was computed using package psych in R software. As we compared the radiomic features from three segmentations (initial P.C, corrected by A.C. and independent F.B.), we used the "ICC2" definition (this measure is one of absolute agreement in the ratings and could be generalize to other observers).

A linear mixed regression model with random intercept was used to evaluate the effect of the multicontrast feature sets, the effect of the choice of classifier, and the effect of additive information from the parenchyma in the feature set on the AUC values (details in supplementary information). T-test was performed for specific pairwise comparison. Analyses were made using R software, v. 3.6.1 (Vienna, Austria).

\section{Pathological examination and response to treatment}

The breast cancer was diagnosed on biopsy. All anatomopathological diagnostics were realized by expert pathologists specialized in breast cancer from our institution. The expression of estrogen receptor, progesterone receptors, and HER-2 status was determined on histopathological pretherapeutic biopsy samples. Hormone receptor negative status was defined if less than $10 \%$ of cells revealed staining for estrogen and progesterone receptors. The expression of HER-2 was considered negative if lower than $1+$ in immunohistochemistry. Tumors with a score at $2+$ required additional in situ hybridization to determine the amplification or non-amplification of HER-2. After a 6-month

217 NAC treatment, all patients underwent breast surgical intervention (lumpectomy or mastectomy). The complete pathological response was defined by the absence of invasive residual tumor in the resected 
stage was ypT0 ypNO or ypTis ypNO, according to 2012 seventh editions of the AJCC Cancer 221 Staging Manual 2012[28].

\section{RESULTS}

\section{Clinical characteristics}

225 Among 79 patients eligible for study enrolment, four patients were excluded: one for MRI-protocol 226 performed one week after the treatment initiation, and three because the SUB3 images were missing or 227 not complete after the data transfer from the archive server. Among the 75 patients enrolled, 11 228 patients presented two lesions, and 3 patients presented three lesions. Two patients presented bilateral 229 tumors. Lesions were analyzed independently. 14 out of these 92 tumors had a clip. Three lesions were 230 excluded for inadequate size to perform radiomic processing. Tumor characteristics are summarized in

\section{$231 \quad$ Table 2.}

2328 out of the 75 patients underwent MRI protocol using the SENSE-Breast-7 coil, and the remaining 233 patients the SENSE-Breast16M. No significant difference in signal-to-noise ratio was observed 234 between images acquisitions with the SENSE-Breast-7 and the SENSE-Breast16M coils (t-test, p235 value $=0.33$ ). For some patient, images from the pre-therapeutic MRI images were missing; details are 236 summarized in Table 3 and reported the proportion of pCR and non-pCR.

\section{Comparison of multi-contrast imaging features}

239 Out of the 89 tumors analyzed, 70 used the whole MRI protocol (T1W, T2W, DWI, and DCE). 240 Multiple combinations of feature set were tested to evaluate the necessity of multiple contrasts 241 imaging (Figure 3). Moreover, different classifiers were used and their performances were compared.

242 Based on the AUC in the training set, the SVM with a quadratic kernel showed the best results (mean 243 AUC of 0.99, Figure 3J). Random Forest and Multilayer perceptron also provided very good results 244 with a mean AUC of 0.93 and 0.90, respectively, for the training set. The "SVM order 1" classifier 245 showed the smallest difference in AUC between the training and test sets reflecting the smallest 246 overfitting (Figure 3 C, F, I, L), or largest bias.

247 The random forest, "SVM order 2", and the MLP classifiers suggest advantages in adding information 248 from different images issued from different MRI sequences. The most interesting configuration of 
feature set appeared to be the combination of DWI, T1W, and T2W features: firstly, the AUC in the validation set was larger than in other configurations even considering the configuration with only T1W features $(+0.034, \mathrm{p}<0.001$, linear mixed model with Dunnett post-hoc test, details in supplementary information); secondly, the overfitting expressed by the difference in AUCs was in the same range than that of the "SVM order $1 "(+0.008, \mathrm{p}=0.564, \mathrm{t}$-test $)$.

The same parameters were used without any characteristics of parenchyma while keeping selected the 20 most relevant parameters. A significant decreased in AUC for the test set was observed for "SVM order 2" (- 0.047, p <0.001, linear mixed model with Dunnett post-hoc test, details in supplementary information). These results showed that the characteristics of both parenchyma and tumors seemed to allow a better prediction of pCR. Unfortunately, the AUC results depended on the repartition of the data in the training set and in test set, and the ranges of AUCs in the test set were large.

260 To go further, it would be interesting to accurately identify the non-pCR patients to avoid exposure to ineffective systemic treatment. In that case, the specificity of the predictive model was the most important. We observed that sensitivity was higher than specificity in our series (Figure 4).

\section{Effect of features selected}

265 The "SVM order 2" combined with DWI, T1, and T2 features seemed to better predict pCR. 266 Therefore, we analyzed the effect of the number of features selected before applying classification. The ReliefF method allowed to promptly classify the most independent features (Table 4). The textural features appear more relevant than shape and 1er order textural features (26 textural features against 4 shape feature). The Figure 5 shows: i) an increase in AUC, specificity and sensitivity values from 1 to 9 parameters; ii) a plateau from 9 to 24 parameters; iii) a slight decrease after 24 parameters.

271 The nine most relevant features appeared as sufficient. In relevant shape feature, the one from 272 parenchyma was very interesting because it reflects the proportion of the sane parenchyma against the 273 tumor (the tumor area was removed from the parenchyma delineation in the binary mask). For the 274 T1W without fat suppression, lobules, ducts and bloods vessels appear in lower values than fatty 275 tissue. The features Short-Run Low Gray-level is the distribution of the short homogeneous runs with low grey-levels and appeared to be a relevant textural feature from parenchyma on T1W imaging. 
The variability analyses were done on a subset of 24 lesions. The segmentation approved by the radiologist $\mathrm{AC}$ was considered the reference. A mean DICE coefficient of $0.95 \pm 0.07$ (range of 0.74 1.00) reflecting the concordance between the two radiologists (PC and $\mathrm{AC}$ ). The mean DICE coefficient was $0.84 \pm 0.10$ (range of $0.56-0.99$ ) comparing the delineation approved by AC and FB. The worst result $(\mathrm{DICE}=0.56)$ came from a stack of images where the contrast enhancement was very low and therefore difficult to segment without information on the pathology. The majority of DICE coefficient was over 0.80 . To measure the impact of delineation variation on radiomic features, an ICC was computed for each feature. On the most relevant features highlighted by the ReliefF method in our study, we observed that textural features have a good ICC (mean 0.88 range $0.65-0.98$ ) but the shape features have a worst ICC2 (mean 0.44 range $0.31-0.56$ ). The textural features represented $75 \%$ of the nine most relevant features.

\section{DISCUSSION}

This study shows that multi-contrast MRI-based radiomics allows to predict the neo-adjuvant chemotherapy response in patients with early triple negative breast cancer with good performances. This study also showed that the choice of classifier strongly influences model performances. While SVM with a linear kernel generalized well, probably due to lower variance and higher bias

297 (highlighted by lower AUC in the training set), other non-linear classifiers such as neural network, 298 quadratic-kernel SVM, and Random Forest, increase performances by reducing bias effect (high performances in the training set), and increase variance and therefore overfitting, as indicated by increased AUC differences between dataset. However, the increase in variety of processable data combining different sources of radiomics (multi-contrast MRI) reduced variance effect as evidenced

302 by reduced AUC differences between dataset when radiomics aggregated from different contrasts. Our 303 results showed that the best model (AUROC at 0.83) was obtained with SVM with a quadratic kernel 304 trained from aggregated radiomics extracted from T1, T2, and diffusion weighted images.

305 From a clinical point of view, radiomic signature could help to better predict pathological complete 
response, and enhanced stratification of patients with an excellent prognosis, and patients at high risk

of chemoresistance. In the latter population, systemic treatment combined to the standard NAC (anthracycline/taxane) may be added, such as platinum-based NAC [29], a PARP inhibitor [30], or even an immune-checkpoint-inhibitor. Indeed, the KEYNOTE-522 trial [31] investigating the addition of prembrolizumab to neo-adjuvant standard chemotherapy showed an increase of $64.8 \%$ of

311 pathological complete response, compared with 51.2\% in the placebo control group. Therefore, it 312 seems important to appropriately select patients who may benefit the most from appropriate systemic 313 treatment, highlighting the medical need for these non-invasive stratification methods.

314 The most informative sequences identified were the association of T1/T2 morphological sequences 315 and diffusion sequence. However, these results also depend on the DCE-MRI sequence used for the 316 segmentation of the lesion. Indeed, we observed some pixels in the fat surrounding the tumors in the 317 mask registered on T1W images; which would not have been integrated if the segmentation had been 318 directly performed on T1W images. The parenchyma features appeared to be relevant in this study. 319 The shape feature computed on the parenchyma binary mask (the difference between the parenchyma 320 and the tumor delineation) appeared to be more relevant than the shape feature computed on tumor binary mask. More generally, the most relevant features were high order of textural features. These textural features would reflect the textural complexity from a macroscopic point of view but the tumor heterogeneity in a microscopy point view. Moreover, the textural features appeared to be more robust to inter-observer variability (different delineation) with high intraclass correlation coefficient.

Our results are consistent with previous studies showing that AUC ranged from 0.67 to 0.87 [1012,14], allow to predict the response to breast cancer chemotherapy. Among these studies, Braman and collaborators showed that the triple negative subgroups had the best results with a 0.93 AUC, and Liu and colleagues reported AUC at 0.86 with fewer TN patients. Our series showed that $64 \%$ of the patients achieved a pathological complete response; this rate is higher than that reported in previous studies with $31 \%$ of patients complete pathological responses in [32].

332 Our study has some limitations. First, the size of our population was limited to 75 patients and the multiparametric radiomic analyses were only achieved in 70 lesions. However, to the best of our knowledge, this TN cohort was the largest cohort dedicated to radiomic studies. The lesion 
segmentation was done manually, which may introduce a degree of subjectivity. In addition, this study was performed in a single institution study; and extrapolating results to population of another center is limited. Lastly, MRI protocols are not standardized between centers in the field of breast cancer analyses inducing variability in the radiomic feature sets. Several studies have shown the sensitivity of radiomic parameters to machine's change or reconstruction parameters [33-35].

Those results have to be confirmed in an external validation cohort with multicentric and prospective data, and more patients.

To conclude, our results confirm that MRI-based radiomics may be relevant to predict neoadjuvant 344 chemotherapy response in early triple negative breast cancer. In addition, our results highlight the 345 interest of using multi-contrast MRI as sources of radiomics to improve model generalization thanks to 346 increased information variety.

\section{Authors' Contribution}

349 Nemeth: Study conception and design, Analysis and interpretation of data, Drafting of 350 manuscript, Critical revision

351 Chaudet: Study conception and design, Acquisition of data, Analysis and interpretation of 352 data, Drafting of manuscript

353 Leporq: Study conception and design, Analysis and interpretation of data, Critical revision

354 Heudel: Study conception and design, Acquisition of data

355 Barabas: Acquisition of data, Analysis and interpretation of data

356 Tredan: Study conception and design

357 Treilleux: Study conception and design

358 Coulon: Study conception and design, Acquisition of data, Analysis and interpretation of data, 359 Drafting of manuscript

360 Pilleul: Analysis and interpretation of data, Critical revision

361 Beuf: Analysis and interpretation of data, Critical revision

\section{Declarations}


364 Ethical approval was waived by the local Ethics Committee of our institution in view of the 365 retrospective nature of the study and all the procedures being performed were part of the 366 routine care.

\section{REFERENCES}

1. Ferlay J, Colombet M, Soerjomataram I, Mathers C, Parkin DM, Piñeros M, Znaor A, Bray F (2018) Estimating the global cancer incidence and mortality in 2018: GLOBOCAN sources and methods. Int J Cancer ijc.31937.

372 2. Billar JAY, Dueck AC, Stucky C-CH, Gray RJ, Wasif N, Northfelt DW, McCullough AE, Pockaj 373 BA (2010) Triple-Negative Breast Cancers: Unique Clinical Presentations and Outcomes. Ann Surg 374 Oncol 17:384-390.

375 3. Foulkes WD, Smith IE, Reis-Filho JS (2010) Triple-Negative Breast Cancer. New England Journal of Medicine 363:1938-1948.

377 4. Derks MGM, van de Velde CJH (2018) Neoadjuvant chemotherapy in breast cancer: more than just 378 downsizing. The Lancet Oncology 19:2-3.

379 5. Kong X, Moran MS, Zhang N, Haffty B, Yang Q (2011) Meta-analysis confirms achieving 380 pathological complete response after neoadjuvant chemotherapy predicts favourable prognosis for 381 breast cancer patients. European Journal of Cancer 47:2084-2090.

382 6. Liedtke C, Mazouni C, Hess KR, André F, Tordai A, Mejia JA, Symmans WF, Gonzalez-Angulo 383 AM, Hennessy B, Green M, Cristofanilli M, Hortobagyi GN, Pusztai L (2008) Response to 384 Neoadjuvant Therapy and Long-Term Survival in Patients With Triple-Negative Breast Cancer. JCO $385 \quad 26: 1275-1281$.

386 7. Earl H, Provenzano E, Abraham J, Dunn J, Vallier A-L, Gounaris I, Hiller L (2015) Neoadjuvant 387 trials in early breast cancer: pathological response at surgery and correlation to longer term outcomes 388 - what does it all mean? BMC Med 13:234.

389 8. for the European Society of Breast Imaging (EUSOBI), with language review by Europa Donna390 The European Breast Cancer Coalition, Mann RM, Balleyguier C, Baltzer PA, Bick U, Colin C, 391 Cornford E, Evans A, Fallenberg E, Forrai G, Fuchsjäger MH, Gilbert FJ, Helbich TH, Heywang- 
RM, Pinker-Domenig K, Skaane P, Sardanelli F (2015) Breast MRI: EUSOBI recommendations for women's information. Eur Radiol 25:3669-3678.

395 9. Lambin P, Rios-Velazquez E, Leijenaar R, Carvalho S, van Stiphout RGPM, Granton P, Zegers 396 CML, Gillies R, Boellard R, Dekker A, Aerts HJWL (2012) Radiomics: Extracting more information 397 from medical images using advanced feature analysis. European Journal of Cancer 48:441-446.

398 10. Liu Z, Li Z, Qu J, Zhang R, Zhou X, Li L, Sun K, Tang Z, Jiang H, Li H, Xiong Q, Ding Y, Zhao 399 X, Wang K, Liu Z, Tian J (2019) Radiomics of Multiparametric MRI for Pretreatment Prediction of 400 Pathologic Complete Response to Neoadjuvant Chemotherapy in Breast Cancer: A Multicenter Study. $401 \quad$ Clin Cancer Res 25:3538-3547.

402 11. Chamming's F, Ueno Y, Ferré R, Kao E, Jannot A-S, Chong J, Omeroglu A, Mesurolle B, 403 Reinhold C, Gallix B (2018) Features from Computerized Texture Analysis of Breast Cancers at 404 Pretreatment MR Imaging Are Associated with Response to Neoadjuvant Chemotherapy. Radiology 405 286:412-420.

12. Fan M, Wu G, Cheng H, Zhang J, Shao G, Li L (2017) Radiomic analysis of DCE-MRI for prediction of response to neoadjuvant chemotherapy in breast cancer patients. European Journal of Radiology 94:140-147.

409 13. Weber JJ, Jochelson MS, Eaton A, Zabor EC, Barrio AV, Gemignani ML, Pilewskie M, Van Zee 410 KJ, Morrow M, El-Tamer M (2017) MRI and Prediction of Pathologic Complete Response in the 411 Breast and Axilla after Neoadjuvant Chemotherapy for Breast Cancer. Journal of the American 412 College of Surgeons 225:740-746.

413 14. Braman NM, Etesami M, Prasanna P, Dubchuk C, Gilmore H, Tiwari P, Plecha D, Madabhushi A 414 (2017) Intratumoral and peritumoral radiomics for the pretreatment prediction of pathological 415 complete response to neoadjuvant chemotherapy based on breast DCE-MRI. Breast Cancer Res 19:57.

416 15. Lambin P, Leijenaar RTH, Deist TM, Peerlings J, de Jong EEC, van Timmeren J, Sanduleanu S, 417 Larue RTHM, Even AJG, Jochems A, van Wijk Y, Woodruff H, van Soest J, Lustberg T, Roelofs E, 418 van Elmpt W, Dekker A, Mottaghy FM, Wildberger JE, Walsh S (2017) Radiomics: the bridge 419 between medical imaging and personalized medicine. Nat Rev Clin Oncol 14:749-762.

420 16. Gillies RJ, Kinahan PE, Hricak H (2016) Radiomics: Images Are More than Pictures, They Are 
E, Ferté C (2017) Promises and challenges for the implementation of computational medical imaging (radiomics) in oncology. Annals of Oncology 28:1191-1206.

19. Agner SC, Rosen MA, Englander S, Tomaszewski JE, Feldman MD, Zhang P, Mies C, Schnall

MD, Madabhushi A (2014) Computerized Image Analysis for Identifying Triple-Negative Breast

Cancers and Differentiating Them from Other Molecular Subtypes of Breast Cancer on Dynamic Contrast-enhanced MR Images: A Feasibility Study. Radiology 272:91-99.

20. Quail DF, Joyce JA (2013) Microenvironmental regulation of tumor progression and metastasis.

433 Nat Med 19:1423-1437.

434 21. Collewet G, Strzelecki M, Mariette F (2004) Influence of MRI acquisition protocols and image intensity normalization methods on texture classification. Magnetic Resonance Imaging 22:81-91.

22. Suk T, Flusser J (2003) Combined blur and affine moment invariants and their use in pattern recognition. Pattern Recognition 36:2895-2907.

23. Ming-Kuei Hu (1962) Visual pattern recognition by moment invariants. IEEE Trans Inform Theory 8:179-187.

24. Tahmasbi A, Saki F, Shokouhi SB (2011) Classification of benign and malignant masses based on

441 Zernike moments. Computers in Biology and Medicine 41:726-735.

25. Saki F, Tahmasbi A, Soltanian-Zadeh H, Shokouhi SB (2013) Fast opposite weight learning rules with application in breast cancer diagnosis. Computers in Biology and Medicine 43:32-41.

444 26. Aerts HJWL, Velazquez ER, Leijenaar RTH, Parmar C, Grossmann P, Carvalho S, Bussink J, 445 Monshouwer R, Haibe-Kains B, Rietveld D, Hoebers F, Rietbergen MM, Leemans CR, Dekker A, 446 Quackenbush J, Gillies RJ, Lambin P (2014) Decoding tumour phenotype by noninvasive imaging 447 using a quantitative radiomics approach. Nat Commun 5:4006.

448 27. Kononenko I, Simec E, Robnik-Sikonja M Overcoming the myopia of inductive learning 449 algorithms with RELIEFF. 17. 
Manual and Handbook, 2nd ed. doi: 10.1007/978-1-4614-2080-4

29. Poggio F, Bruzzone M, Ceppi M, Pondé NF, La Valle G, Del Mastro L, de Azambuja E,

Lambertini M (2018) Platinum-based neoadjuvant chemotherapy in triple-negative breast cancer: a systematic review and meta-analysis. Annals of Oncology 29:1497-1508.

30. Loibl S, O’Shaughnessy J, Untch M, Sikov WM, Rugo HS, McKee MD, Huober J, Golshan M, von Minckwitz G, Maag D, Sullivan D, Wolmark N, McIntyre K, Ponce Lorenzo JJ, Metzger Filho O, Rastogi P, Symmans WF, Liu X, Geyer CE (2018) Addition of the PARP inhibitor veliparib plus carboplatin or carboplatin alone to standard neoadjuvant chemotherapy in triple-negative breast cancer (BrighTNess): a randomised, phase 3 trial. The Lancet Oncology 19:497-509. Aktan G, Dent R, O’Shaughnessy J, KEYNOTE-522 Investigators (2020) Pembrolizumab for Early Triple-Negative Breast Cancer. N Engl J Med 382:810-821.

32. Houssami N, Macaskill P, von Minckwitz G, Marinovich ML, Mamounas E (2012) Meta-analysis of the association of breast cancer subtype and pathologic complete response to neoadjuvant chemotherapy. European Journal of Cancer 48:3342-3354.

33. Yan J, Chu-Shern JL, Loi HY, Khor LK, Sinha AK, Quek ST, Tham IWK, Townsend D (2015) Impact of Image Reconstruction Settings on Texture Features in 18F-FDG PET. Journal of Nuclear Medicine 56:1667-1673. Gur RC, Gur RE, Schultz RT, Verma R, Shinohara RT (2017) Harmonization of multi-site diffusion 473 tensor imaging data. NeuroImage 161:149-170.

474 35. Reuzé S, Orlhac F, Chargari C, Nioche C, Limkin E, Riet F, Escande A, Haie-Meder C, Dercle L, 475 Gouy S, Buvat I, Deutsch E, Robert C (2017) Prediction of cervical cancer recurrence using textural 476 features extracted from 18F-FDG PET images acquired with different scanners. Oncotarget. doi: 


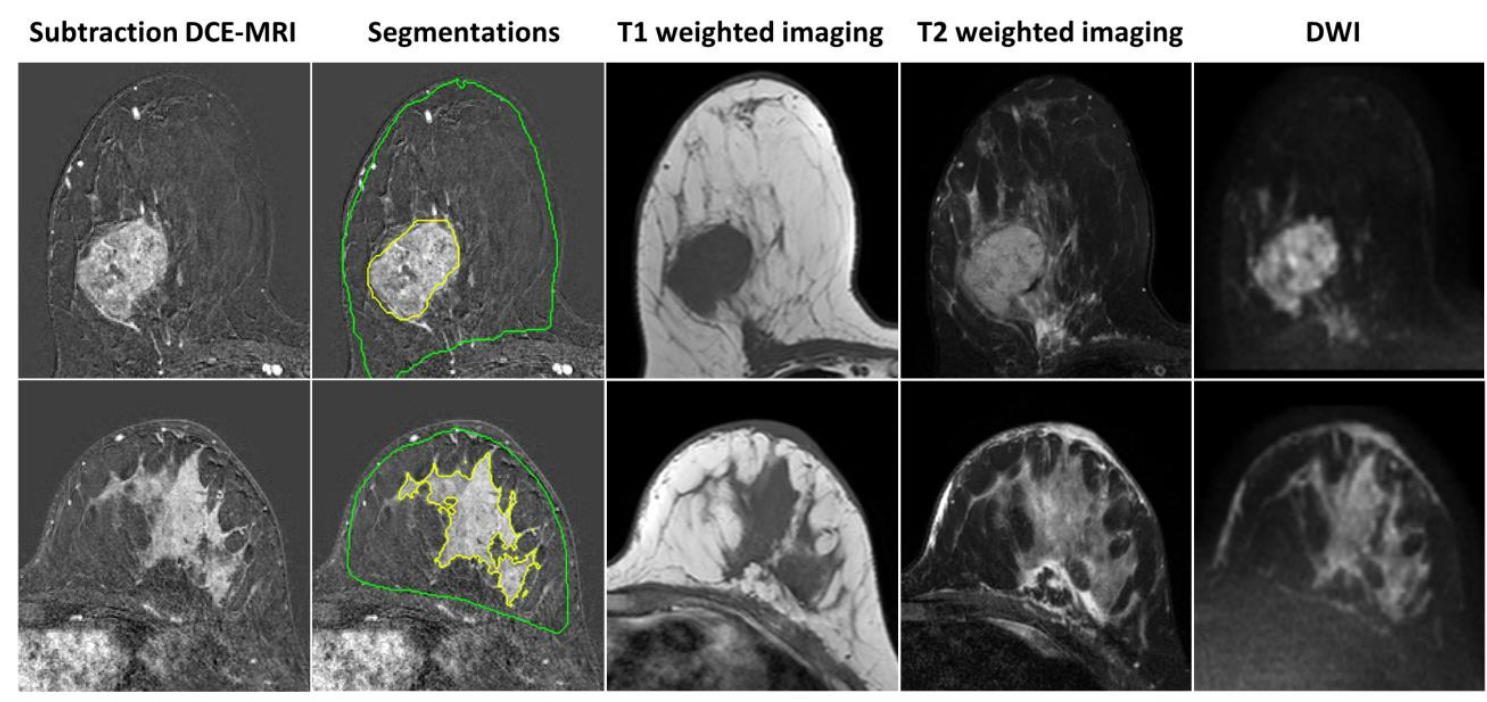

480 Figure 1. Two triple-negative breast cancers imaged with pre-therapeutic MRI protocol. First

481 line: a mass tumor is shown; second line: a non-mass tumor is illustrated. Segmentation of tumor (in

482 yellow) and segmentation of parenchyma (in green excluding tumor area) were made on derived DCE-

483 MRI. For non-mass tumor, the option "snake" of ITK-SNAP was used to perform the segmentation. 


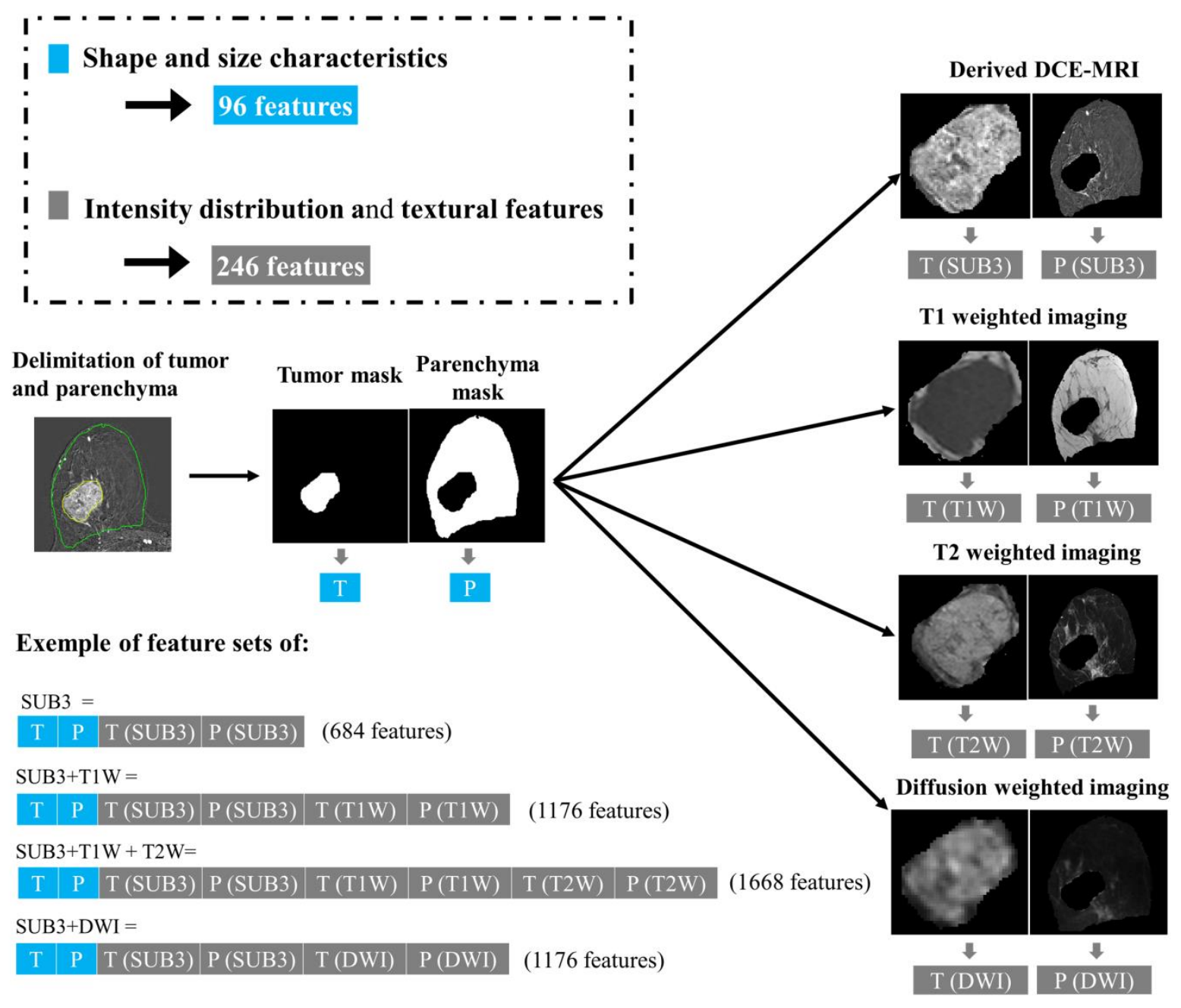

487 Figure 2. Pipeline of radiomic feature extraction and combination of feature sets. 


\section{SVM order 1}

A

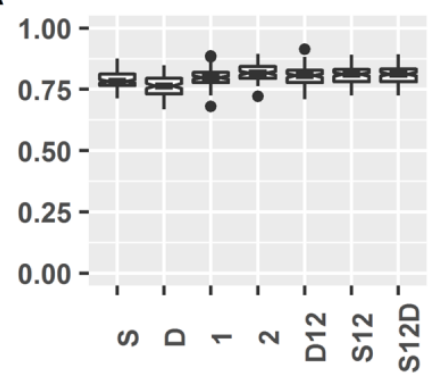

D

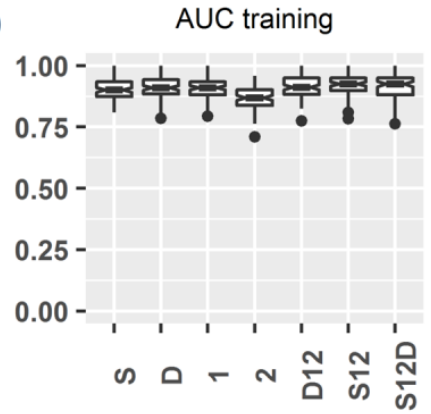

G

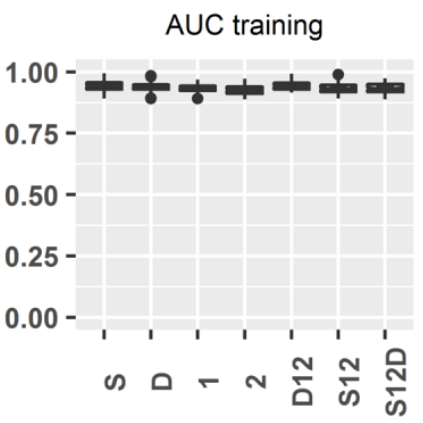

J

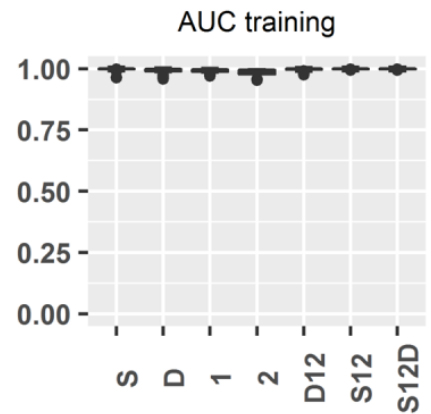

B

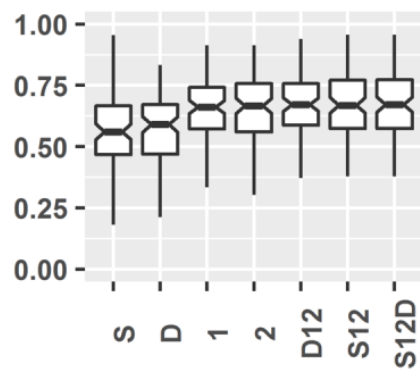

Multilayer perceptron

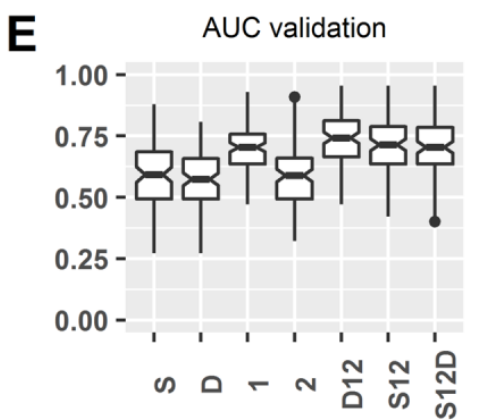

\section{Random Forest}

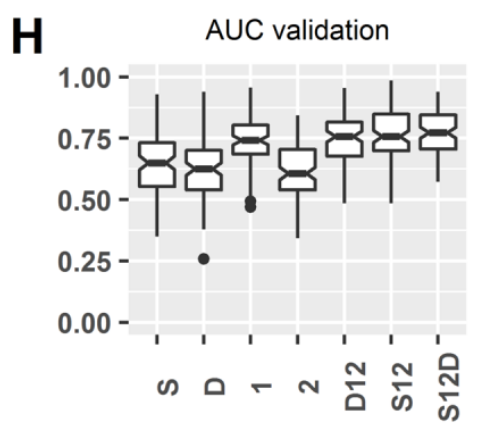

\section{SVM order 2}

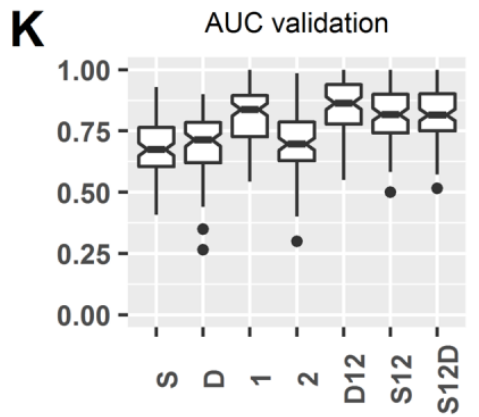

C Difference of AUCs

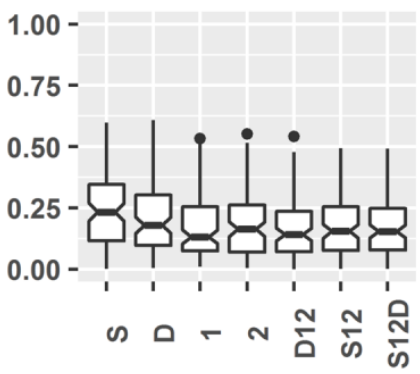

$F$

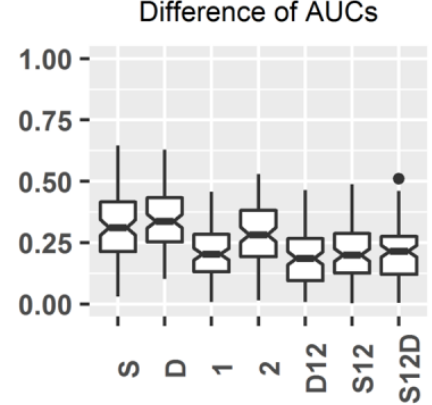

I

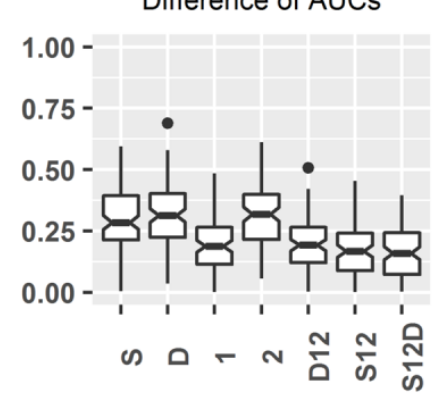

L

Difference of AUCs

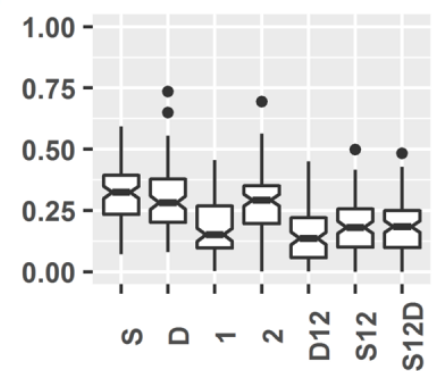

489 Figure 3. Boxplots of different configurations of radiomic feature set. AUC: area under the curve

490 ROC for the training set and for the test set. For the case "S" only features from the SUB3 were

491 included in the feature set; " $D$ " corresponds to the diffusion weighted imaging, "1" corresponds to the

492 T1-weighted imaging and "2" corresponds to the T2-weighted imaging. The results of all possible

493 configurations are presented as supplementary information. 


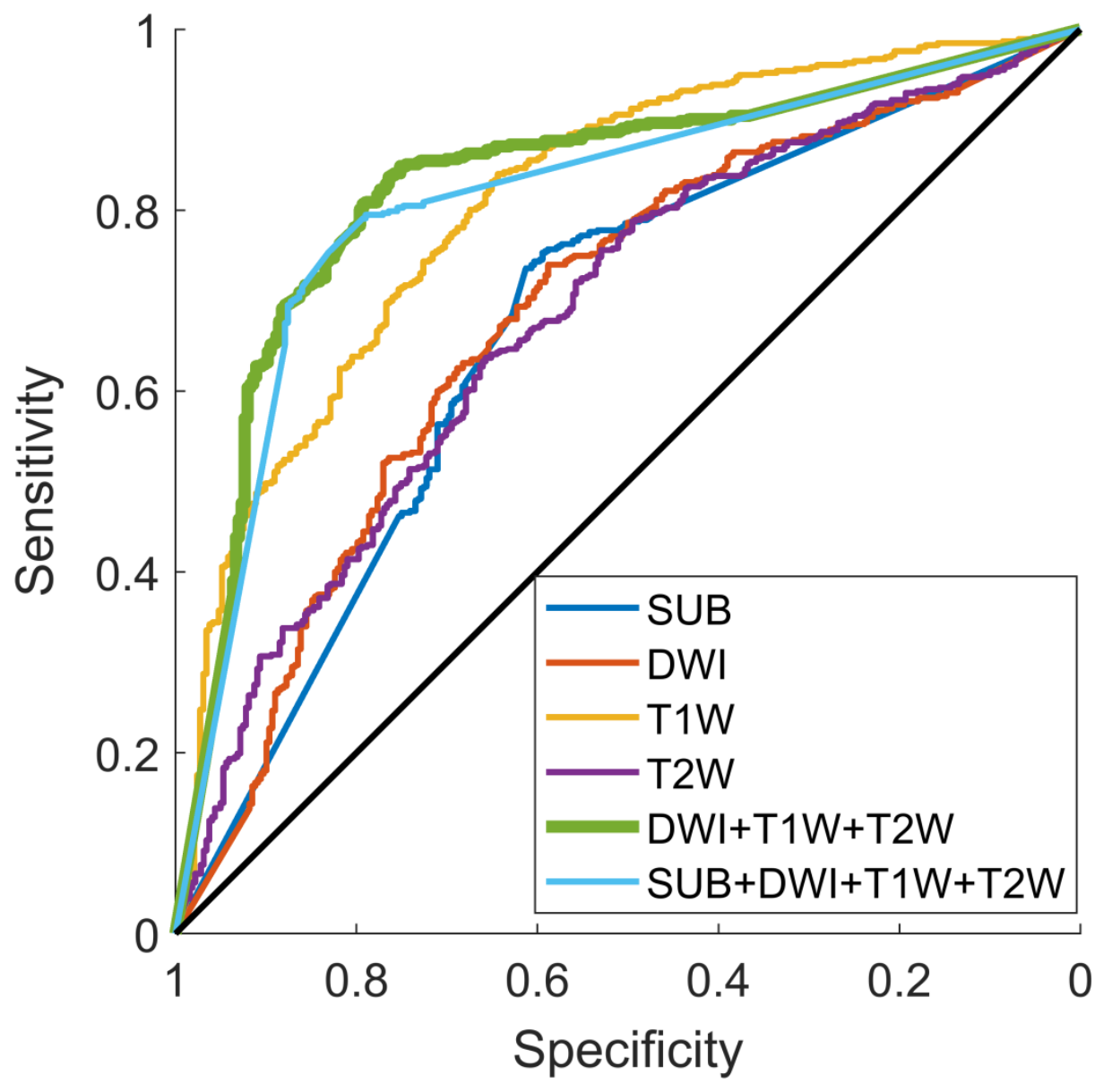

494

495 Figure 4: ROC curves for the SVM classifier with quadratic kernel. The most interesting

496 configuration of feature set appeared to be the combination of DWI, T1W and T2W features with $497 \quad$ AUC $=0.83$, sensitivity $=0.85$ and specificity $=0.75$. 

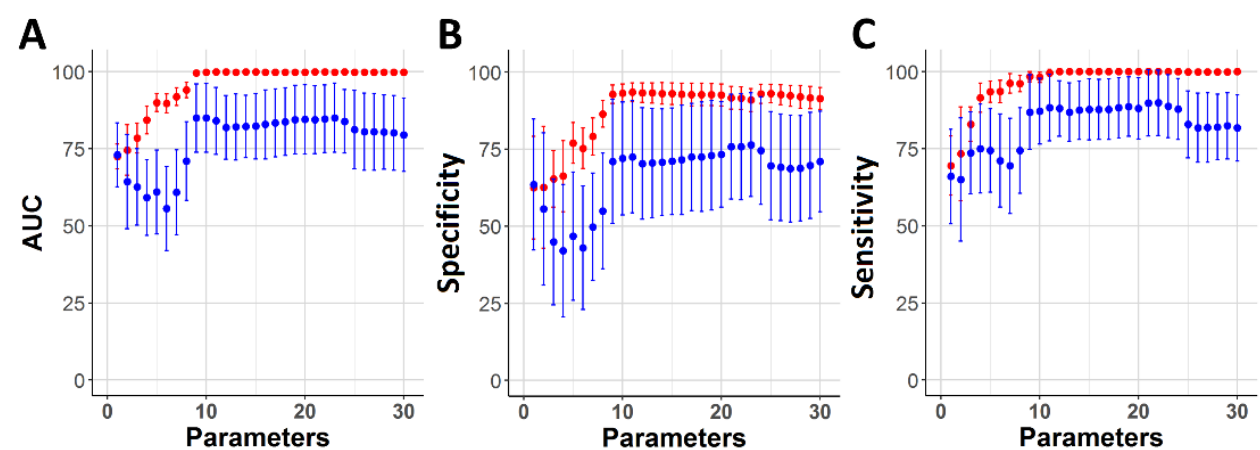

\section{Training sets Validation sets}

501 Figure 5: Variation of model performances in training and test sets according to the number of 502 feature selected with the ReliefF method. 
Table 1: Summary of sequence parameters of T1W (T1-weigthed imaging), T2W (T2-weighted imaging), DWI (diffusion weighted imaging) and DCE (dynamic contrast enhancement).

\begin{tabular}{|c|c|c|c|c|}
\hline & T1W & T2W & DWI & DCE \\
\hline Pulse-sequence & TSE & SPAIR (TSE) & $\mathrm{SE}$ & TRIVE (TFE) \\
\hline Flip angle & $90^{\circ}$ & $90^{\circ}$ & $90^{\circ}$ & $12^{\circ}$ \\
\hline TE & $7 \mathrm{~ms}$ & $70 \mathrm{~ms}$ & $75 \mathrm{~ms}$ & $2.73 \mathrm{~ms}$ \\
\hline TR & $600 \mathrm{~ms}$ & $2.8 \mathrm{~s}$ & $3.5 \mathrm{~s}$ & $5.5 \mathrm{~ms}$ \\
\hline Slice thickness & 3 or $3.5 \mathrm{~mm}$ & 3 or $3.5 \mathrm{~mm}$ & $2 \mathrm{~mm}$ & $2 \mathrm{~mm}$ \\
\hline Pixel resolution & $0.45 \times 0.45 \mathrm{~mm}^{2}$ & $0.55 \times 0.55 \mathrm{~mm}^{2}$ & $1.17 \times 1.17 \mathrm{~mm}^{2}$ & $0.75 \times 0.75 \mathrm{~mm}^{2}$ \\
\hline Fat suppression & no & yes & yes & yes \\
\hline $\mathrm{b}\left(\mathrm{s} / \mathrm{mm}^{2}\right)$ & / & / & 700 & / \\
\hline Contrast agent & no & no & no & yes \\
\hline
\end{tabular}

506 
509 Table 2. Patient information and tumor characteristics

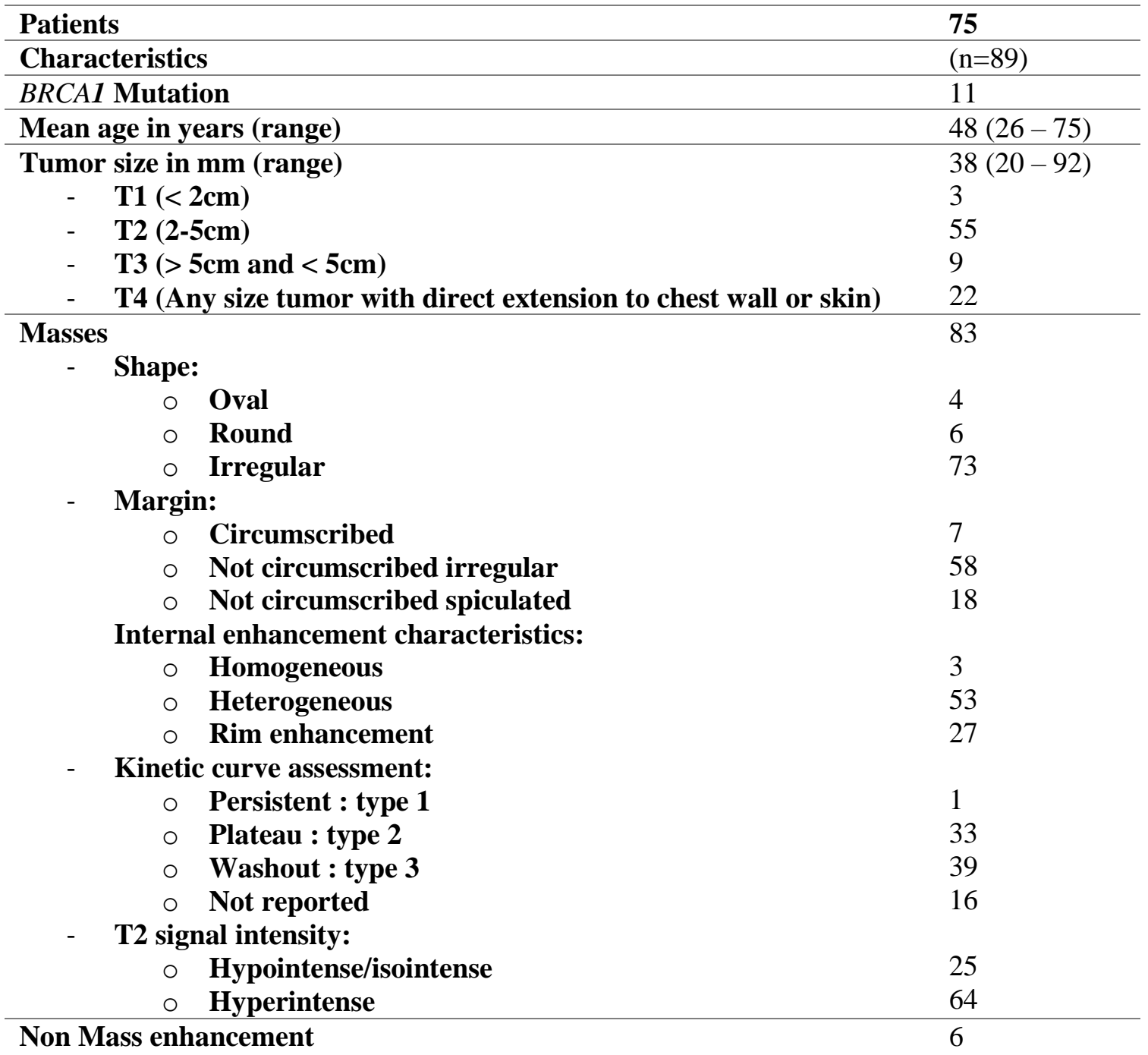

- Distribution:

$\begin{array}{lll}\circ & \text { Focal } & 0 \\ \circ & \text { Linear } & 0 \\ \circ & \text { Segmental } & 2 \\ \circ \text { Regional } & 2 \\ \circ & \text { Multiple regions } & 0 \\ \circ & \text { Diffuse } & 2\end{array}$

- Internal enhancement patterns :

$\begin{array}{lll}\circ & \text { Homogeneous } & 1 \\ \circ & \text { Heterogeneous } & 3 \\ \circ & \text { Clumped } & 2 \\ \circ & \text { Clustered ring } & 0\end{array}$

Adenopathy

- Axillary 53

- Internal mammary 20

Pathologic complete response

- Yes

- No 32

Histologic types

- Infiltrating ductal carcinoma $\quad 78$

- Infiltrating ductal carcinoma + ductal carcinoma in situ 6

Others (metaplastic carcinoma, myoepithelial carcinoma, medullary carcinoma) 
Type of surgery

Lumpectomy

Mastectomy

510

511 
512 Table 3. Number of lesions imaged with DCE-MRI, T1W, T2W, and DWI sequences.

\begin{tabular}{|c|c|c|c|c|}
\hline & $\begin{array}{c}\text { SUB3 } \\
(\mathrm{N}=89)\end{array}$ & $\begin{array}{c}\text { T1W } \\
(\mathrm{N}=86)\end{array}$ & $\begin{array}{c}\text { T2W } \\
(\mathrm{N}=76)\end{array}$ & $\begin{array}{c}\text { DWI } \\
(\mathrm{N}=72)\end{array}$ \\
\hline pCR & 57 & 56 & 47 & 45 \\
\hline non-pCR & 32 & 30 & 29 & 27 \\
\hline
\end{tabular}

513

514 


\begin{tabular}{|c|c|c|c|c|c|}
\hline $\begin{array}{l}\text { Order } \\
\text { (ReliefF) }\end{array}$ & $\begin{array}{l}\text { Type of } \\
\text { tissue }\end{array}$ & $\begin{array}{l}\text { Type of } \\
\text { imaging }\end{array}$ & $\begin{array}{l}\text { Type of } \\
\text { features }\end{array}$ & Function & Parameter names \\
\hline 1 & Tumor & $\mathrm{T} 2 \mathrm{~W}$ & Textural & SURF & std_blobs_strength \\
\hline 2 & Parenchyma & $\begin{array}{l}\text { Binary } \\
\text { mask }\end{array}$ & Shape & $\begin{array}{l}\text { Affine } \\
\text { moment }\end{array}$ & Affine_moment_invariant_6 \\
\hline 3 & Tumor & $\mathrm{T} 2 \mathrm{~W}$ & Textural & Quadtree & sum_blocks \\
\hline 4 & Parenchyma & T1W & Textural & GLRLM & Short_Run_Low_Gray_Level_Emphasis \\
\hline 5 & Tumor & $\mathrm{T} 2 \mathrm{~W}$ & Textural & Quadtree & mean_c \\
\hline 6 & Tumor & $\mathrm{T} 1 \mathrm{~W}$ & Textural & SURF & std_blobs_strength \\
\hline 7 & Tumor & $\begin{array}{l}\text { Binary } \\
\text { mask }\end{array}$ & Shape & $\begin{array}{l}\text { Zernike } \\
\text { moment }\end{array}$ & Zernike_moment_Phi8 \\
\hline 8 & Tumor & $\begin{array}{l}\text { Binary } \\
\text { mask }\end{array}$ & Shape & $\begin{array}{l}\mathrm{Hu} \\
\text { moment }\end{array}$ & Hu_moment_1 \\
\hline 9 & Tumor & T1W & Textural & Gabor & Gabor_square_energy_s1 \\
\hline 10 & Tumor & $\mathrm{T} 2 \mathrm{~W}$ & Textural & Quadtree & mean_r \\
\hline 11 & Tumor & T1W & Textural & Grad & G2_y_sum \\
\hline 12 & Tumor & DWI & Textural & SURF & mean_blobs_strength \\
\hline 13 & Tumor & T1W & Textural & Gabor & Gabor_square_energy_s2 \\
\hline 14 & Tumor & T1W & Textural & Grad & G1_y_sum \\
\hline 15 & Tumor & T1W & Textural & Grad & L4_sum \\
\hline 16 & Tumor & T1W & Textural & Grad & L2_sum \\
\hline 17 & Tumor & T1W & Textural & Grad & L1_y_sum \\
\hline 18 & Tumor & T1W & Textural & Grad & G1_x_sum \\
\hline 19 & Tumor & T1W & Textural & Grad & G2_x_sum \\
\hline 20 & Tumor & T1W & Textural & Grad & L3_sum \\
\hline 21 & Parenchyma & T1W & Textural & GLCM & Information_measure_of_correlation_2 \\
\hline 22 & Tumor & T1W & Textural & Grad & L1_x_sum \\
\hline 23 & Tumor & T1W & Textural & Gabor & Gabor_square_energy_s5 \\
\hline 24 & Tumor & $\begin{array}{l}\text { Binary } \\
\text { mask }\end{array}$ & Shape & $\begin{array}{l}\text { Skelet } \\
\text { features }\end{array}$ & std_2 \\
\hline 25 & Parenchyma & DIFF & Textural & GLCM & Correlation \\
\hline 26 & Tumor & DIFF & Textural & $\begin{array}{l}\text { FFT } \\
\text { features }\end{array}$ & rank_F_orient1 \\
\hline 27 & Tumor & DIFF & Textural & $\begin{array}{l}\text { FFT } \\
\text { features }\end{array}$ & rank_F_orient2 \\
\hline 28 & Tumor & DIFF & Textural & $\begin{array}{l}\text { FFT } \\
\text { features }\end{array}$ & rank_F_orient3 \\
\hline 29 & Tumor & T1W & Textural & Gabor & Gabor_square_energy_s3 \\
\hline 30 & Tumor & $\mathrm{T} 2 \mathrm{~W}$ & Textural & Quadtree & length_quadtree \\
\hline
\end{tabular}




\section{Supplementary information}

520

521

522

523

524

525

526

527

528

529

530

531

532

533

534

535

536

537

538

539

540

541

542

543

544

545

546

547

548

549

550

\section{$\underline{\text { Statistical analyses }}$}

The aim of this supplementary information is to provide complete statistical analyses about the impact of different configuration of feature set on classifier performance. Indeed, in the paper, only the most relevant results were illustrated.

Multiple effects were analyzed:

1. Multiple configurations of feature sets from multi-contrast imaging (ex: combining features from the DWI and T1W).

2. Effect of adding information from parenchyma (combining features from the parenchyma and from the tumor).

3. Effect of the classifier chose.

For each of the 100 random draws of training/validation set (performing with "cvpartition" Matlab function), fifteen configurations of feature set were tested. For each feature set, 4 classifiers were applied to provide a predictive model and the AUC was used to evaluate the performance of each classifier. To compare the fifteen configurations of feature, a linear mixed-effect model (LMM) was performed on AUC of validation set (or AUC of training set) for each classifier using "Imer" $\mathrm{R}$ function. For the design of the LMM, we considered repeated measurements on training/test set (100 different cases) with three fixed factors: Configurations (15 possibilities), Type of initial features set (tumor only or tumor + parenchyma) and Classifier (4 possibilities: SVM order 1, Random Forest, Multilayer perceptron and SVM order 2); and a random effect. The normality of each distribution was validated by a quantile-quantile plot. Figures S1, S2, S3 and S4 shows boxplot of the fifteen possible configurations with data "tumor only" for A,B and C graphs and with data "tumor + parenchyma" for D, E and F. The fixed-effect results of LMM were summarized in table S5.

The LMM demonstrated a significant effect of adding information from the parenchyma with greater values of AUC_training and AUC_validation (mean difference of respectively 0.020 and 0.047 after the correction of "configurations" effect and "classifier" effect). Most of configurations using multi-contrast imaging have higher AUC values than using only one contrast; expect for the configuration "DWI + T2W". The classifier with the highest AUC values in both training and validation sets was the SVM with quadratic kernel. 
SVM order 1 (tumor only)
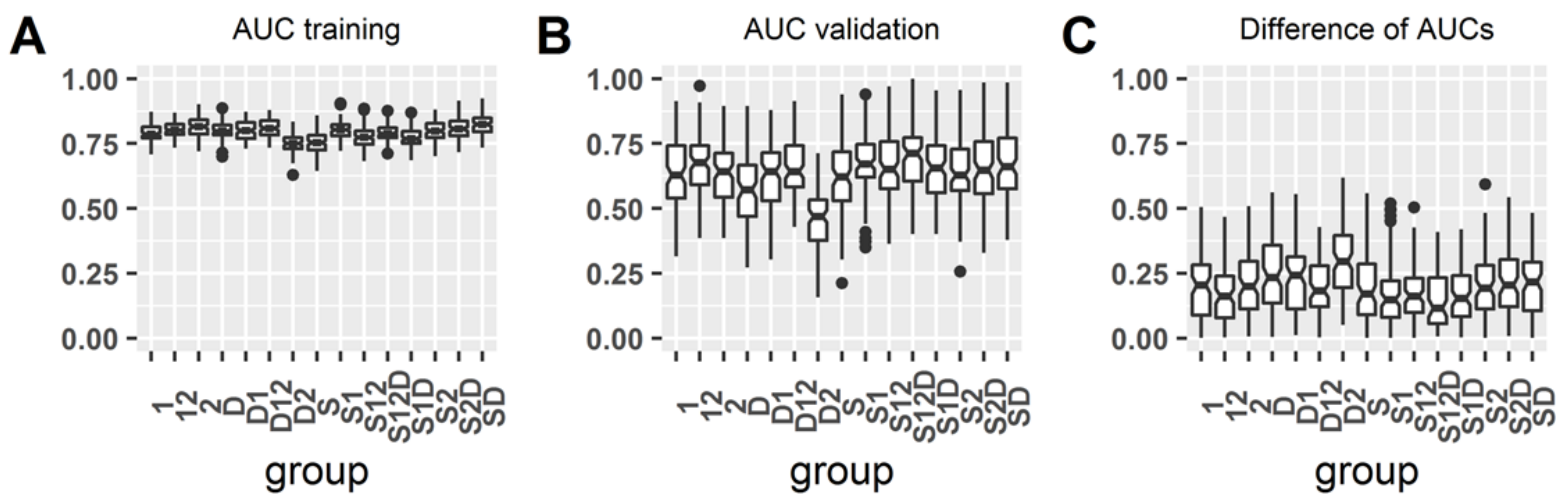

\section{SVM order 1 (tumor + parenchyma)}

D

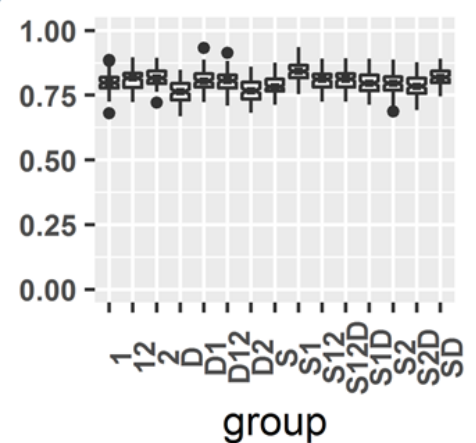

E

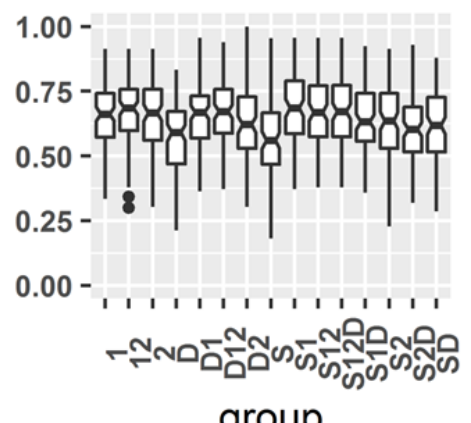

$\mathbf{F}$ Difference of AUCs $1.00-$ $0.75-$

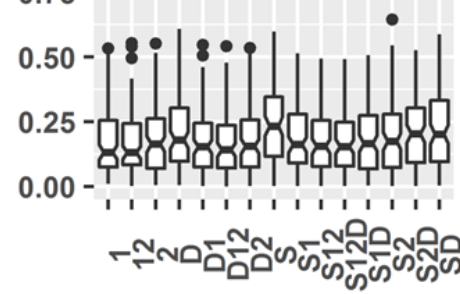

group

552 Figure S1 (Support vector machine with linear kernel): Boxplot of AUC distribution in

553 the training/validation set and the difference of the AUC of the two sets. Feature set used

554 for the classification was designed as follow: "1" designs T1-weighted imaging, "2" for

555 T2-weighted imaging, "D" for diffusion weighted imaging and " $S$ " for the subtraction of

556 DCE-MRI. The initial feature set was composed of tumor features for A, B, C. The

557 initial feature set was composed of tumor and parenchyma features for $D, E$, and $F$. 


\section{Multilayer perceptron (tumor only)}

A

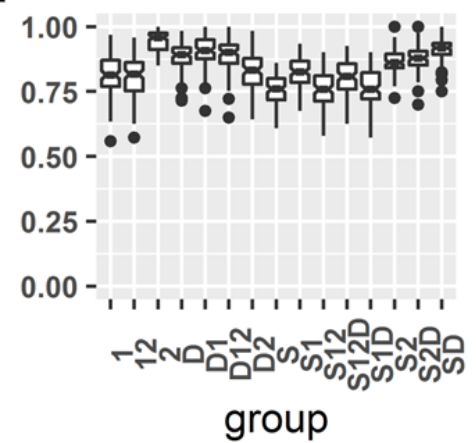

B

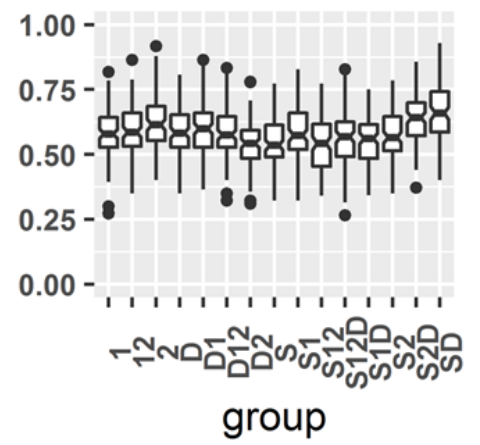

C

$1.00-$

$0.75-$

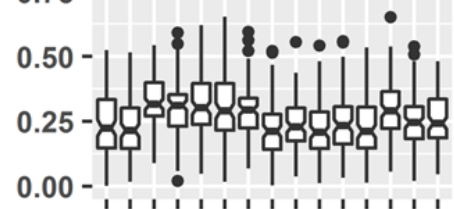

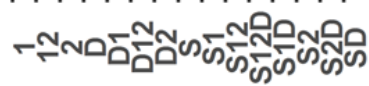

group

\section{Multilayer perceptron (tumor + parenchyma)}

D

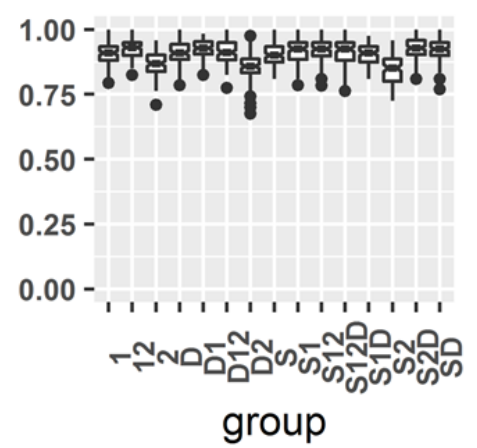

E

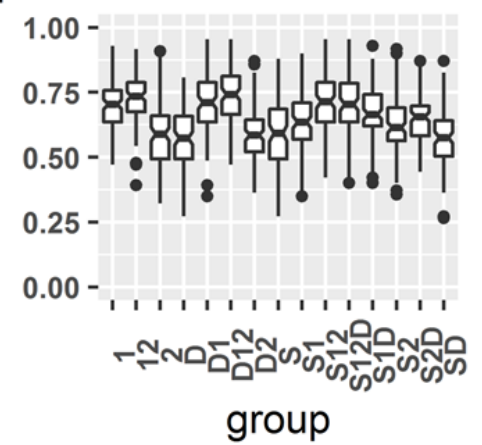

$\mathbf{F}$

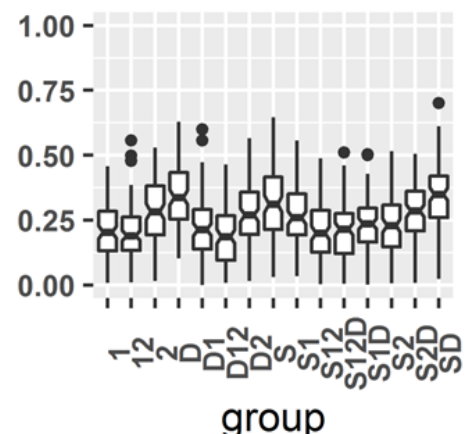

Figure S2 (Multilayer perceptron classifier): Boxplot of AUC distribution in the

561 training/validation set and the difference of the AUC of the two sets. Feature set used for

562 the classification was designed as follow: "1" designs T1-weighted imaging, "2" for T2-

563 weighted imaging, " $D$ " for diffusion weighted imaging and "S" for the subtraction of

564 DCE-MRI. The initial feature set was composed of tumor features for A, B, C. The

565 initial feature set was composed of tumor and parenchyma features for $\mathrm{D}, \mathrm{E}$, and $\mathrm{F}$. 

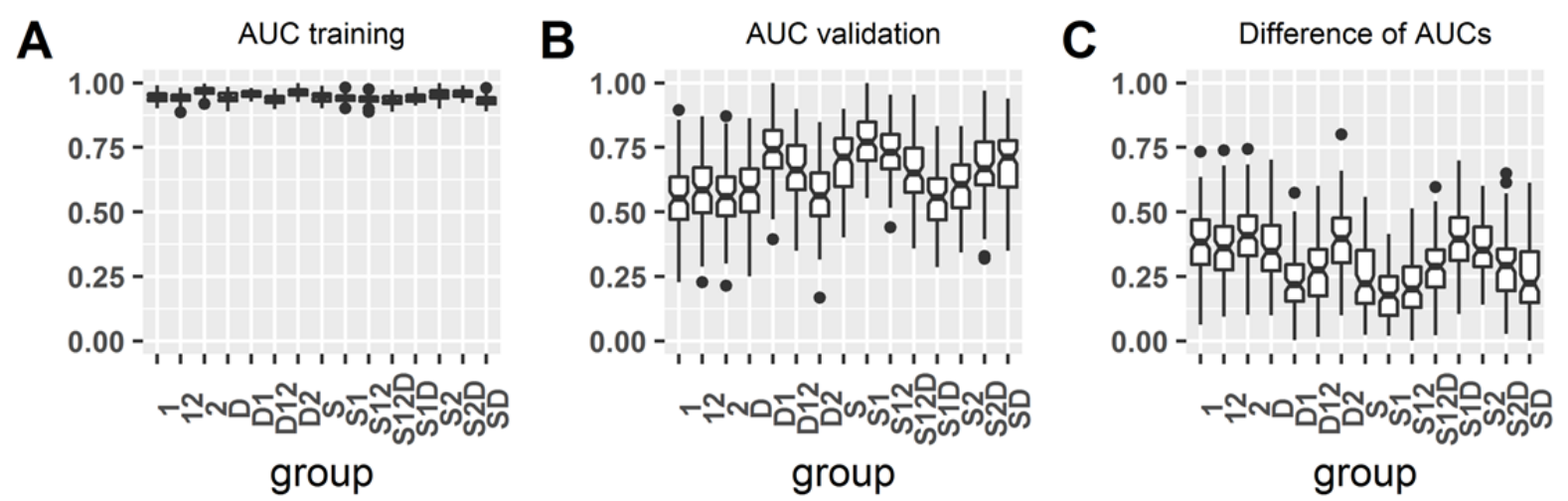

\section{Random Forest (tumor + parenchyma)}

D

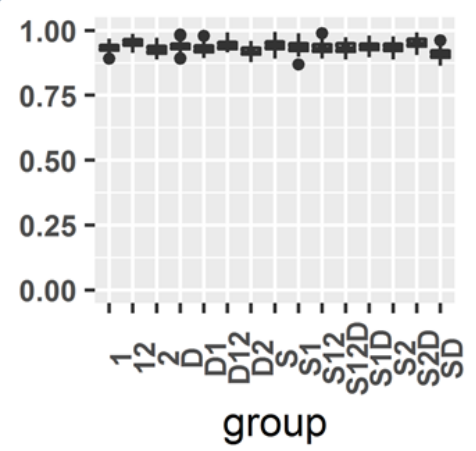

E

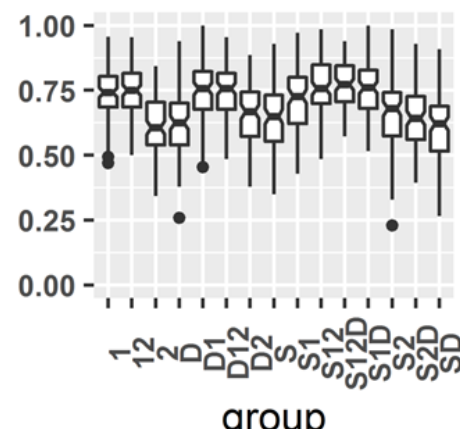

$\mathbf{F}$

Difference of AUCs

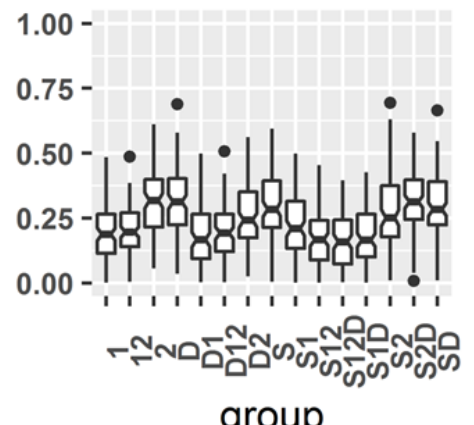

Figure S3 (Random Forest classifier): Boxplot of AUC distribution in the

569 training/validation set and the difference of the AUC of the two sets. Feature set used for

570 the classification was designed as follow: "1" designs T1-weighted imaging, "2" for T2-

571 weighted imaging, " $D$ " for diffusion weighted imaging and "S" for the subtraction of

572 DCE-MRI. The initial feature set was composed of tumor features for A, B, C. The

573 initial feature set was composed of tumor and parenchyma features for $\mathrm{D}, \mathrm{E}$, and $\mathrm{F}$. 
SVM order 2 (tumor only)
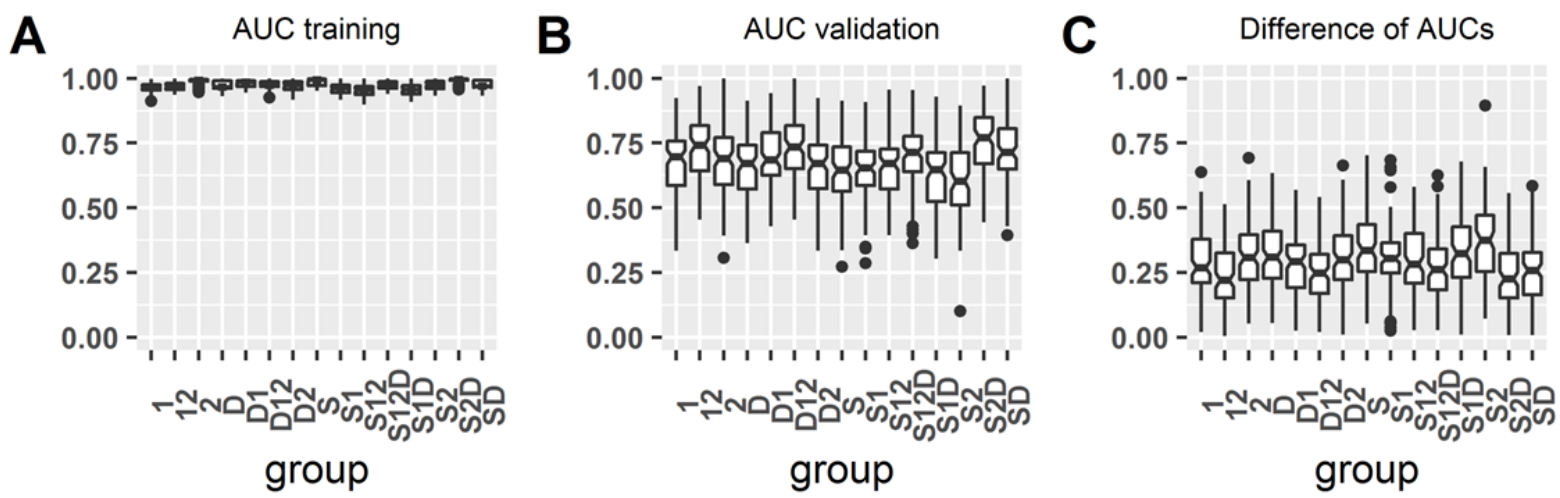

\section{SVM order 2 (tumor + parenchyma)}

D

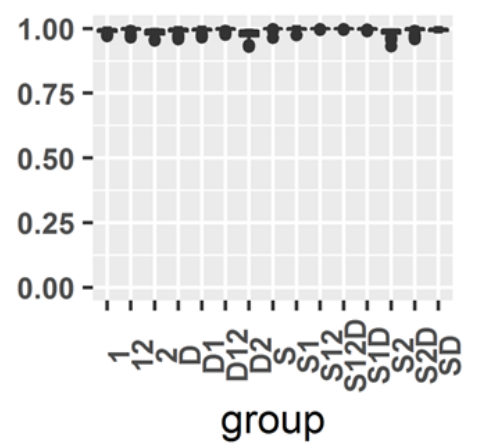

E

AUC validation

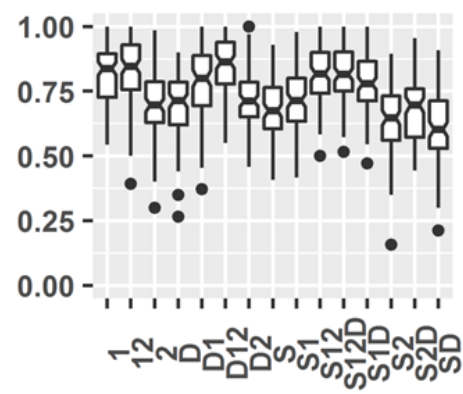

group
$\mathbf{F}$

Difference of AUCs

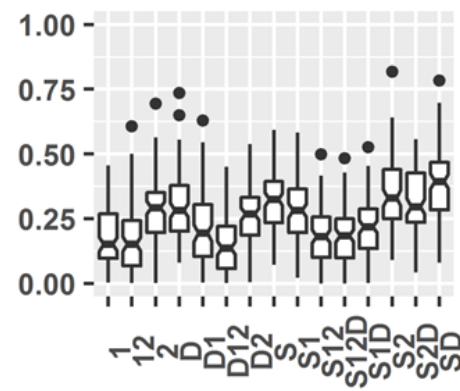

group

Figure S4 (Support vector machine with quadratic kernel): Boxplot of AUC distribution

577 in the training/validation set and the difference of the AUC of the two sets. Feature set 578 used for the classification was designed as follow: "1" designs T1-weighted imaging, "2" 579 for T2-weighted imaging, " $D$ " for diffusion weighted imaging and " $S$ " for the 580 subtraction of DCE-MRI. The initial feature set was composed of tumor features for A, 581 B, C. The initial feature set was composed of tumor and parenchyma features for D, E, 582 and $\mathbf{F}$. 


\begin{tabular}{|c|c|c|}
\hline & \multicolumn{2}{|c|}{ Mean difference from reference } \\
\hline & AUC Training & AUC Validation \\
\hline \multicolumn{3}{|l|}{ Initial feature set } \\
\hline Tumor only (reference) & -- & -- \\
\hline Tumor + parenchyma & $0.020 * * *$ & $0.047 * * *$ \\
\hline \multicolumn{3}{|l|}{ Configurations } \\
\hline Sub3 (reference) & -- & -- \\
\hline $\mathrm{T} 1 \mathrm{~W}$ & $0.008 * * *$ & $0.049 * * *$ \\
\hline $\mathrm{T} 1 \mathrm{~W}+\mathrm{T} 2 \mathrm{~W}$ & $0.016 * * *$ & $0.073 * * *$ \\
\hline $\mathrm{T} 2 \mathrm{~W}$ & $0.030 * * *$ & $0.011(\mathrm{p}=0.502)$ \\
\hline DWI & $0.016 * * *$ & $-0.013(\mathrm{p}=0.252)$ \\
\hline $\mathrm{DWI}+\mathrm{T} 1 \mathrm{~W}$ & $0.028 * * *$ & $0.075 * * *$ \\
\hline $\mathrm{DWI}+\mathrm{T} 1 \mathrm{~W}+\mathrm{T} 2 \mathrm{~W}$ & $0.024 * * *$ & $0.083 * * *$ \\
\hline $\mathrm{DWI}+\mathrm{T} 2 \mathrm{~W}$ & $-0.006(p=0.088)$ & $-0.021(\mathrm{p}=0.005)$ \\
\hline Sub3 +T1W & $0.018 * * *$ & $0.060 * * *$ \\
\hline Sub3 +T1W + T2W & $0.001(\mathrm{p}=1.000)$ & $0.072 * * *$ \\
\hline Sub3 +T1W + T2W + DWI & $0.010 * * *$ & $0.077 * * *$ \\
\hline Sub3 +T1W + DWI & $-0.001(\mathrm{p}=1.000)$ & $0.036 * * *$ \\
\hline Sub3 +T2W & $0.010 * * *$ & $0.003(\mathrm{p}=1.000)$ \\
\hline Sub3 +T2W + DWI & $0.028 * * *$ & $0.040 * * *$ \\
\hline Sub3 + DWI & $0.026 * * *$ & $0.022(\mathrm{p}=0.002)$ \\
\hline \multicolumn{3}{|l|}{ Classifier } \\
\hline SVM order 1 (reference) & -- & -- \\
\hline MLP & $0.075 * * *$ & $-0.019 * * *$ \\
\hline Random Forest & $0.143 * * *$ & $0.031 * * *$ \\
\hline SVM order 2 & $0.186 * * *$ & $0.073 * * *$ \\
\hline
\end{tabular}

